\title{
Impact of delta-doping position on photoluminescence in type-II InAs/GaAsSb quantum dots
}

\author{
Yeongho Kim ${ }^{1}$, Keun-Yong Ban ${ }^{1,3}$, Darius Kuciauskas ${ }^{2}$, Patricia C. Dippo ${ }^{2}$ and Christiana B. Honsberg ${ }^{1}$ \\ ${ }^{1}$ Department of Electrical Engineering, Arizona State University, Tempe, AZ 85287-5706, USA \\ ${ }^{2}$ National Renewable Energy Laboratory, Golden, Colorado 80401, USA \\ E-mail: ykim172@asu.edu
}

We studied the optical properties of InAs/ $\mathrm{GaAs}_{0.83} \mathrm{Sb}_{0.17}$ quantum dots (QDs), with varying silicon deltadoping position (spatial distance, $d=0.5,1$, and $2 \mathrm{~nm}$ ), using photoluminescence (PL) measurements. Compared with the undoped QDs, the PL peak energies of the ground state (GS) emissions for the doped QDs with $d=0.5$ and $2 \mathrm{~nm}$ were found to be greatly blueshifted by $\sim 31 \mathrm{meV}$, which was much larger than that for the doped QDs with $d=1 \mathrm{~nm}$. The radiative recombination rate of the GS emissions for the doped QDs with $d=1 \mathrm{~nm}$ was estimated to be slower than that for the other doped QDs at $10 \mathrm{~K}$. The doped QDs with $d=1 \mathrm{~nm}$ showed the fastest redshift of the GS peak energy with temperature and lowest thermal activation energy $(151 \mathrm{meV})$ of electrons among the QD samples. Further, the time-resolved PL data revealed that the average carrier lifetime (6.3 ns) in the doped QDs with $d=1 \mathrm{~nm}$ was longer even than that in the undoped QDs (5.5 ns) because of the weakened electron-hole wavefunction overlap by the V-shaped potential barrier in the doped QDs.

\section{Introduction}

Third generation solar cells using nanostructures, such as intermediate band solar cells (IBSCs) and multiple exciton generation solar cells, are of interest for overcoming the Shockley-Queisser efficiency limit for conventional single-junction solar cells $[1,2]$. In IBSCs, the InAs quantum dots (QDs) are incorporated in the intrinsic layer of a $p-i-n$ solar cell to improve photocurrent generation by sub-bandgap photon absorption [1]. Therefore, InAs/GaAs QD systems have been extensively studied for IBSC application [1-3]. However, the performance of the fabricated IBSCs has been much poorer than predicted by theoretical models. Improved device performance has not been achieved despite the detection of small photocurrent signals thought to arise from subband absorptions in the near infrared wavelength region [4]. The degradation in the device performance is mainly caused by reduction in the open-circuit voltage $\left(\mathrm{V}_{\mathrm{oc}}\right)$ due to a large valence band offset (VBO) [5] and crystalline defects, such as threading dislocations and interface defects, generated by the strain accumulation during QD growth [6].

Recently, InAs/GaAsSb QDs have been suggested as an alternative to InAs/GaAs QDs because their band alignment, QD size, and areal density can be tuned by varying the $\mathrm{Sb}$ composition $[7,8]$. To efficiently realize IBSCs using InAs/GaAsSb QDs, QD electronic levels corresponding to the intermediate band (IB) should be half-filled with electrons in order to balance sub-bandgap transitions from the valence band (VB) to the IB and from the IB to the conduction band (CB). This helps to boost photocurrent generation, and thereby the energy conversion efficiency of the IBSCs. In this study, we applied silicon $(\mathrm{Si})$ delta $(\delta)$-doping beneath the QD plane to supply electrons into QD electronic levels [1,9]. The optical properties of the Si doped QDs are significantly affected by the doping parameters such as doping density and doping position [10,11]. In particular, the doping position should be well calibrated to precisely control the electron occupation at the QD sub-bands because it causes substantial change in interband optical transitions and carrier lifetimes.

In this article, we discuss the optical characteristics of type-II InAs/GaAsSb QDs with Si $\delta$-doped in different positions. Our results indicate that the QD structure with $\delta$-doping located $1 \mathrm{~nm}$ below the InAs QD plane experiences similar interband optical transitions and prolonged carrier lifetimes at low temperatures as

${ }^{3}$ Present address: Applied Materials, Inc., Santa Clara, CA 95054-3299, USA. 
compared to the undoped QDs. InAs/GaAsSb QDs with these characteristics can be employed as a promising absorber of sub-band gap photons for IBSCs.

\section{Experimental procedure}

The InAs/GaAsSb QD samples were grown by solid-source molecular beam epitaxy (MBE) on semi-insulating GaAs (001) substrates. After thermal cleaning of the substrate, a $400 \mathrm{~nm}$ thick GaAs buffer layer was grown at $600{ }^{\circ} \mathrm{C}$. Subsequently, a $10 \mathrm{~nm}$ thick $\mathrm{GaAs}_{0.83} \mathrm{Sb}_{0.17}$ barrier layer was grown at $500{ }^{\circ} \mathrm{C}$. The InAs with a nominal coverage of two monolayers was deposited on the GaAsSb and covered with a $10 \mathrm{~nm}$ thick $\mathrm{GaAs}_{0.83} \mathrm{Sb}_{0.17}$ layer. QD formation was confirmed by in-situ reflection high-energy electron diffraction (RHEED) patterns. The Si $\delta$-doping with an areal density of $2 \times 10^{12} \mathrm{~cm}^{-2}$ was introduced in the GaAsSb layer, below the InAs layer, to inject free electrons into the QD plane $(d=0.5,1$, and $2 \mathrm{~nm})$. Finally, the QD structures were capped with a $200 \mathrm{~nm}$ thick GaAs layer at $600^{\circ} \mathrm{C}$. The doped QD samples with $d=2,1$, and $0.5 \mathrm{~nm}$ are referred to as QD2, QD3, and QD4, respectively. An undoped QD sample (QD1) was also grown under the same growth conditions without $\delta$-doping as a reference.

For continuous-wave (cw) photoluminescence (PL) measurements, the as-grown samples were excited by an Nd:YAG laser $\left(\lambda_{\text {exc }}=532 \mathrm{~nm}\right)$, and the PL spectra were recorded using a standard lock-in detection technique. In addition, carrier dynamics in the QDs was investigated using time-resolved PL (TRPL) spectra measured at $4.2 \mathrm{~K}$ using a time-correlated single photon counting system with a $200 \mathrm{fs}$ Ti:Sapphire laser $\left(\lambda_{\mathrm{exc}}=\right.$ $800 \mathrm{~nm})$.

\section{Results and discussion}

To examine the interband transitions of type-II InAs/GaAsSb QDs, cw-PL measurements were taken at an excitation power of $100 \mathrm{~mW}$. Figure 1 shows that the PL intensity of QD1 (without Si $\delta$-doping) is much stronger than that of the doped QDs. The PL intensity quenching in the doped QDs can be explained as follows. The each QD of the doped samples has $\sim 60$ electrons positioned at its sub-band energy levels through the $\delta$-doping. Such a high density of electrons per dot is sufficient for strong carrier-carrier scattering leading to Auger-type nonradiative recombination [12]. Additionally, the PL intensities may also be reduced because of Shockley-Read-Hall (SRH) recombination facilitated by the point and planar defects, such as Ga vacancy $\left(\mathrm{V}_{\mathrm{Ga}}\right)$ and $\mathrm{Si}-\mathrm{X}$ defects $\left(\mathrm{As}_{\mathrm{Ga}}-\mathrm{Si}_{\mathrm{As}}-\mathrm{V}_{\mathrm{Ga}}\right)$, formed during the $\delta$-doping process [13].

The PL peak energies of the ground state (GS) emissions were centered at 1.103, 1.137, 1.110, and $1.131 \mathrm{eV}$ for QD1, QD2, QD3, and QD4, respectively. The GS peaks of the doped samples were blueshifted with respect to that of the undoped sample due to the band bending effect caused by $\delta$-doping. As more electrons populate QD electronic levels, the local electric field induced by the spatial separation of the electrons and holes is increased, leading to strong band bending [14]. Compared to the GS peak of QD1, the GS peak of QD3 $(d=1$ $\mathrm{nm}$ ) was negligibly blueshifted by $7 \mathrm{meV}$ whereas those of QD2 and QD4 were largely blueshifted by 34 and 28 meV, respectively. In case of QD4 $(d=0.5 \mathrm{~nm})$ where the $\delta$-doping position is the closest to the QD plane, the electrons of the $\delta$-doped plane can easily tunnel through the thinner barrier into the QD plane. To quantitatively investigate the electron tunneling effect, the tunneling probabilities of electrons at the ground state in the $\delta$-doped plane into the QD plane are calculated by solving Poisson's and time-independent Schrödinger equations. It is assumed for simplicity that the potential barrier between the $\delta$-doped plane and the QD plane is a square barrier with a potential barrier of $\sim 500 \mathrm{meV}$, which is approximate conduction band offset at an InAs/GaAsSb heterojunction. The ground state energy $\left(\mathrm{E}_{0}\right)$ of electrons in the $\delta$-doped plane is determined to be $48.2 \mathrm{meV}$ given by [15]

$$
E_{0}=\frac{3}{10}\left(\frac{81}{16} \frac{e^{4} \hbar^{2} n^{2}}{\varepsilon^{2} m^{*}}\right)^{1 / 3}
$$

where $\mathrm{n}, \varepsilon$, and $\mathrm{m}^{*}$ are a $\delta$-doping density, a permittivity of GaAsSb, and an effective mass of electron, respectively. The tunneling probability (T) of electrons to the QD plane is calculated using an equation below. 


$$
T=\left[1+\frac{1}{4}\left[\frac{U^{2}}{E_{0}\left(U-E_{0}\right)}\right]\left[\frac{e^{\alpha d}+e^{-\alpha d}}{2}\right]^{2}\right]^{-1}
$$

where $U$ is a potential barrier $(500 \mathrm{meV}), \mathrm{E}_{0}$ is an electron energy at the ground state $(48.2 \mathrm{meV})$, $\alpha=\sqrt{2 m^{*}\left(U-E_{0}\right)} / \hbar$, and $\mathrm{d}$ is a potential barrier width $(0.5,1$, and $2 \mathrm{~nm})$. The evaluated tunneling probabilities are $22.3,14.7$, and $3.6 \%$ for the $\delta$-doped QD samples with $d=0.5,1$, and $2 \mathrm{~nm}$, respectively. Hence, more electrons reside in the QD potential well due to the highest tunneling probability for QD4 ( $d=0.5 \mathrm{~nm})$.

Unlike the undoped QDs, the PL emissions of the doped samples exhibit another excited state (ES) peak of $\mathrm{E}_{1} \mathrm{H}_{2}$, which was observed at 1.256, 1.219, and 1.235 eV for QD2, QD3, and QD4, respectively. This ES peak is due to the state filling effect of the QDs. The state filling effect is typically observed when the lower sub-band levels are fully occupied by the increased electron population.

Figure 2(a) shows the integrated PL intensities of the GS emissions as a function of the excitation power ranging from 1 to $100 \mathrm{~mW}$ at $10 \mathrm{~K}$. The integrated PL intensity linearly increases with excitation power on a log-log scale. This relation can be described as [16]

$$
\mathrm{I}_{\mathrm{PL}}=\eta \mathrm{P}^{\mathrm{k}}
$$

where $\mathrm{I}_{\mathrm{PL}}$ is the integrated PL intensity, $\eta$ is the emission efficiency, $\mathrm{P}$ is the excitation power, and the exponent $\mathrm{k}$ depends on the radiative recombination mechanism. The exponents, given by the slopes of the fitted lines, were determined to be 1.03, 0.64, 0.69, and 0.78 for QD1, QD2, QD3, and QD4, respectively. The strict linear dependence of the integrated PL intensity on the excitation power observed for QD1 is due to the bound exciton recombination in the QDs. On the other hand, the doped QDs display sub-linear behaviors $(\mathrm{k}<1)$ with low PL efficiencies [17] owing to the Auger-type and/or SRH recombination induced by introducing Si $\delta$-doping.

In figure 2(b), the GS peak energies of the QD emissions are plotted against the cube root of the excitation power. The PL peak energies of all studied samples increased linearly with increasing $P^{l / 3}$. This blueshift behavior indicates type-II band alignment, in which the electrons and holes are spatially separated. For the excitation power range of $1 \mathrm{~mW}$ to $100 \mathrm{~mW}$, the blueshifts of the GS emissions were 30.4, 10.5, 24.4, and $13.8 \mathrm{meV}$ for QD1, QD2, QD3, and QD4, respectively. The blueshift of the GS emissions for type-II band structures can be expressed by [14]

$$
\Delta E_{C} \propto\left(\frac{P}{\gamma}\right)^{1 / 3}
$$

where $\mathrm{P}$ and $\gamma$ are the excitation power density and radiative recombination rate, respectively. The stronger blueshift for QD1 indicates that the radiative recombination rate in the undoped QDs is slower than that in the doped QDs, as suggested by equation (4). In addition, it is estimated that the $\gamma$ of QD2 and QD4 ( $d=2$ and $0.5 \mathrm{~nm})$ is faster than that of QD3 $(d=1 \mathrm{~nm})$. Compared to QD3 $(d=1 \mathrm{~nm})$, the photoexcited carriers can be more efficiently transported and captured into the QDs in QD2 and QD4 $(d=2$ and $0.5 \mathrm{~nm})$. Such a high carrier population in the QDs produces a strong Coulombic interaction and Auger-type recombination, leading to the fast carrier relaxation between the intersubband levels and thus fast radiative recombination for the doped QDs $(d=2$ and $0.5 \mathrm{~nm})$. On the other hand, the low hole injection into the InAs/GaAsSb interface for QD3 $(d=1 \mathrm{~nm})$ by the $\mathrm{V}$-shaped potential barrier in the VB leads to the slow radiative recombination which is the reason for the large PL blueshift of $24.4 \mathrm{meV}$.

Figure 3(a) depicts the temperature dependence of the GS peaks for the QD samples. The PL peak energy was found to decrease with increasing temperature because of the carrier redistribution in the QDs by the carrier thermalization and interdot hopping process, through which carriers hop from smaller QDs to larger ones as temperature increases, in accordance with the empirical Varshni law [18]. The GS peak energy for all the QD samples redshifted at a greater rate with increasing temperature as compared to the InAs bulk. Using the Varshni model for data fitting, the redshift rates were evaluated to be $0.30,0.29,0.45$, and $0.29 \mathrm{meV} / \mathrm{K}$ for QD1, QD2, QD3, and QD4, respectively. Interestingly, the GS peak energy of QD3 is redshifted at a higher rate with increasing temperature than those of the other QD samples.

To explain the temperature dependence of the GS peak energy shift, we estimated the thermal activation energies of the electrons confined in the QDs by fitting the integrated PL intensities over temperature with an Arrhenius equation [9], as shown in figure 3(b). In the high-temperature regime, the activation energies were found to be 167, 227, 151, and $240 \mathrm{meV}$ for QD1, QD2, QD3, and QD4, respectively. The reason why the 
QD3 $(d=1 \mathrm{~nm})$ has the smallest activation energy of $151 \mathrm{meV}$ is as follows. It should be noted that the high carrier population in the QDs causes the carriers to be more strongly bound by the enhanced Coulombic interaction, suppressing the thermal escape of carriers and, in turn, increasing the thermal activation energy [9]. The hole transport of QD3 $(d=1 \mathrm{~nm})$ can be hindered through the V-shaped potential barrier in the VB induced by the $\delta$-doping. Thus, the wavefunction overlap of the electrons and holes is weakened for QD3 $(d=1 \mathrm{~nm})$, indicating that it has a smaller thermal activation energy than the other samples. The thermal activation energy corresponds to the transition energy required for the electrons to escape from the ground state to the continuum state in the CB of the QDs. Because low thermal activation energy of the electrons expedites the interdot hopping process [19], the QD3, which has the smallest activation energy among all samples, shows the fastest GS redshift with increasing temperature as seen in figure 3(a).

To corroborate the trend observed in the cw-PL results, we performed TRPL measurements for all the samples. Figure 4 exhibits the time evolution of the GS emissions measured at $4.2 \mathrm{~K}$. The temporal decay traces are fitted with a biexponential decay function:

$$
I(t)=A_{1} \exp \left(-t / \tau_{1}\right)+A_{2} \exp \left(-t / \tau_{2}\right)
$$

where $\mathrm{I}$ is the integrated PL intensity, $\mathrm{A}_{1(2)}$ is an amplitude constant, and $\tau_{1(2)}$ is the carrier lifetime. The average carrier lifetime is defined as

$$
\tau_{\text {avg }}=\frac{\sum A_{i} \tau_{i}}{\sum A_{i}}
$$

The values for carrier lifetimes $\tau_{1}, \tau_{2}$, and $\tau_{\text {avg }}$ and the amplitude ratios $\mathrm{A}_{2} / \mathrm{A}_{1}$ are summarized in table 1 . The amplitude ratios of the doped QDs are smaller than that of the undoped QDs. This implies that nonradiative recombination is stronger in the doped QDs because of Auger-type and/or SRH nonradiative recombination [20].

The $\tau_{\text {avg }}$ of QD2 $(d=2 \mathrm{~nm})$ is shorter than that of QD1 as shown in table 1. This is attributed to increased electron population of QD electronic levels injected from the $\delta$-doping. Thus, more carrier-carrier interaction occurs leading to fast relaxation and radiative recombination compared to the undoped QDs. As the position of the $\delta$-doped plane is varied from $d=2$ to $d=1 \mathrm{~nm}$, the hole transport towards the InAs/GaAsSb interface after optical excitation is constrained by the $\mathrm{V}$-shaped potential barrier in the VB formed through the $\delta$ doping as seen in figure 5. This gives rise to a low electron-hole binding energy and hence weak Coulombic interaction of carriers. Accordingly, the extended spatial separation of carriers decreases radiative recombination rate, leading to increasing the $\tau_{\text {avg }}$ of $6.3 \mathrm{~ns}$ for QD3. In addition, when the $\delta$-doped plane is very closely positioned beneath the QD plane $(d=0.5 \mathrm{~nm})$ the $\mathrm{V}$-shaped potential well in the CB is highly overlapped with the QD potential as shown in figure 5. This enhances the Coulombic interaction of carriers although hole transport is suppressed by the V-shaped potential barrier in the VB. Such high electron population by tunneling and the potential well overlap is responsible for the shortest $\tau_{\text {avg }}$ of $2.1 \mathrm{~ns}$ for QD4. Further, it is plausible to expect that the InAs QDs can be directly doped with Si atoms through the interdiffusion of them into the QDs during the InGa intermixing between the InAs QDs and the surrounding GaAsSb matrix. Dao et al. reported that the TRPL carrier lifetime in the $\operatorname{In}_{0.2} \mathrm{Ga}_{0.8} \mathrm{As} / \mathrm{GaAs}$ quantum well (QW), in which $\mathrm{Si} \delta$-doping was placed in the center of the QW, was shorter than that of the undoped QW owing to ionized impurity scattering and interface scattering [21]. Therefore, we have demonstrated that the optimization of the doping position can help prolong carrier lifetimes as well as control the electron injection at the sub-bands in the QDs, both of which are key parameters for high efficiency IBSCs.

\section{Conclusion}

In conclusion, the optical properties of the $\mathrm{Si} \delta$-doped InAs/GaAsSb QDs with different doping positions were investigated. Compared to the doped QDs $(d=2$ and $0.5 \mathrm{~nm})$, the PL peak position of GS emission for the doped QDs $(d=1 \mathrm{~nm})$ was significantly less blueshifted and the radiative recombination rate was expected to be slower at $10 \mathrm{~K}$ as a result of reduced hole transport towards the QD plane by the V-shaped potential barrier in the VB. Further, the blocked hole transport in the doped QDs $(d=1 \mathrm{~nm})$ suppressed the Coulombic interaction of carriers which reduces the thermal activation energy of them and thereby facilitates the Varshni-like PL redshift with increasing temperature. Lastly, the improved carrier lifetime was obtained for the doped QDs $(d=1$ $\mathrm{nm}$ ) due to the weak electron-hole wavefunction overlap by the potential barrier effect. The experimental results 
in this work demonstrate that the carrier lifetime in the QDs can be improved without significant change in the interband optical transitions by optimizing the $\delta$-doping position.

\section{Acknowledgments}

This material is based upon work primarily supported by the National Science Foundation (NSF) and the Department of Energy (DOE) under NSF CA No. EEC-1041895. Any opinions, findings and conclusions or recommendations expressed in this material are those of the author(s) and do not necessarily reflect those of NSF or DOE. Additionally, this work was supported by the National Renewable Energy Laboratory as a part of the Non-Proprietary Partnering Program under Contract No. De-AC36-08-GO28308 with the U.S. Department of Energy. 


\section{References}

[1] A. Martı', E. Antolı'n, C. R. Stanley, C. D. Farmer, N. Lo'pez, P. Di'az, E. Ca'novas, P. G. Linares and A. Luque 2006 Phys. Rev. Lett. 97247701

[2] W.-S. Liu, H.-M. Wu, F.-H. Tsao, T.-L. Hsu and J.-I. Chyi 2012 Sol. Energ. Mat. Sol. 105237

[3] A. Luque and A. Marti 1997 Phys. Rev. Lett. 785014

[4] D. Zhou, G. Sharma, S. F. Thomassen, T. W. Reenaas and B. O. Fimland 2010 Appl. Phys. Lett. 96061913

[5] A. Luque and A. Mart1' 2010 Adv. Mater. 22160

[6] S. P. Bremner, K.-Y. Ban, N. N. Faleev, C. B. Honsberg and D. J. Smith 2013 J. Appl. Phys. 114103511

[7] K.-Y. Ban, D. Kuciauskas, S. P. Bremner and C. B. Honsberg 2012 J. Appl. Phys. 111104302

[8] K.-Y. Ban, S. P. Bremner, G. Liu, S. N. Dahal, P. C. Dippo, A. G. Norman and C. B. Honsberg 2010 Appl. Phys. Lett. 96 183101

[9] K.-Y. Ban, W.-K. Hong, S. P. Bremner, S. N. Dahal, H. McFelea and C. B. Honsberg 2011 J. Appl. Phys. 109014312

[10] J. Phillips, K. Kamath, X. Zhou, N. Chervela and P. Bhattacharya 1997 Appl. Phys. Lett. 712079

[11] H. Khmissi, M. Baira, L. Sfaxi, L. Bouzaïene, F. Saidi, C. Bru-Chevallier and H. Maaref 2011 J. Appl. Phys. 109054316

[12] Y. D. Jang, T. J. Badcock, D. J. Mowbray, M. S. Skolnick, J. Park, D. Lee, H. Y. Liu, M. Hopkinson, R. A. Hogg and A. D. Andreev 2008 Appl. Phys. Lett. 93101903

[13] R. C. Newman, M. J. Ashwin, M. R. Fahy, L. Hart, S. N. Holmes, C. Roberts, X. Zhang and J. Wagner 1996 Phys. Rev. B. 548769

[14] Y. S. Chiu, M. H. Ya, W. S. Su and Y. F. Chen 2002 J. Appl. Phys. 925810

[15] E. F. Schubert 1990 J. Vac. Sci. Technol. A, 82980

[16] T. Schmidt, K. Lischka and W. Zulehner 1992 Phys. Rev. B 458989

[17] J. L. Casas Espínola, T. V. Torchynska, E. Velasquez Lozada, L. V. Shcherbyna, A. Stintz, R. Peña Sierra 2007 Physica B 401-402584

[18] Y. P. Varshni 1967 Physica 34149

[19] D. Alonso-Álvarez, A. G. Taboada, J. M. Ripalda, B. Alén, Y. González, L. González, J. M. García, F. Briones, A. Martí, A. Luque, A. M. Sánchez and S. I. Molina 2008 Appl. Phys. Lett. 93123114

[20] Ü. Özgür, Y. Fu, Y. T. Moon, F. Yun, H. Morkoç and H. O. Everitt 2005 J. Appl. Phys. 97103704

[21] L. V. Dao, M. Gal, G. Li and C. Jagadish 2000 J. Appl. Phys. 873896 


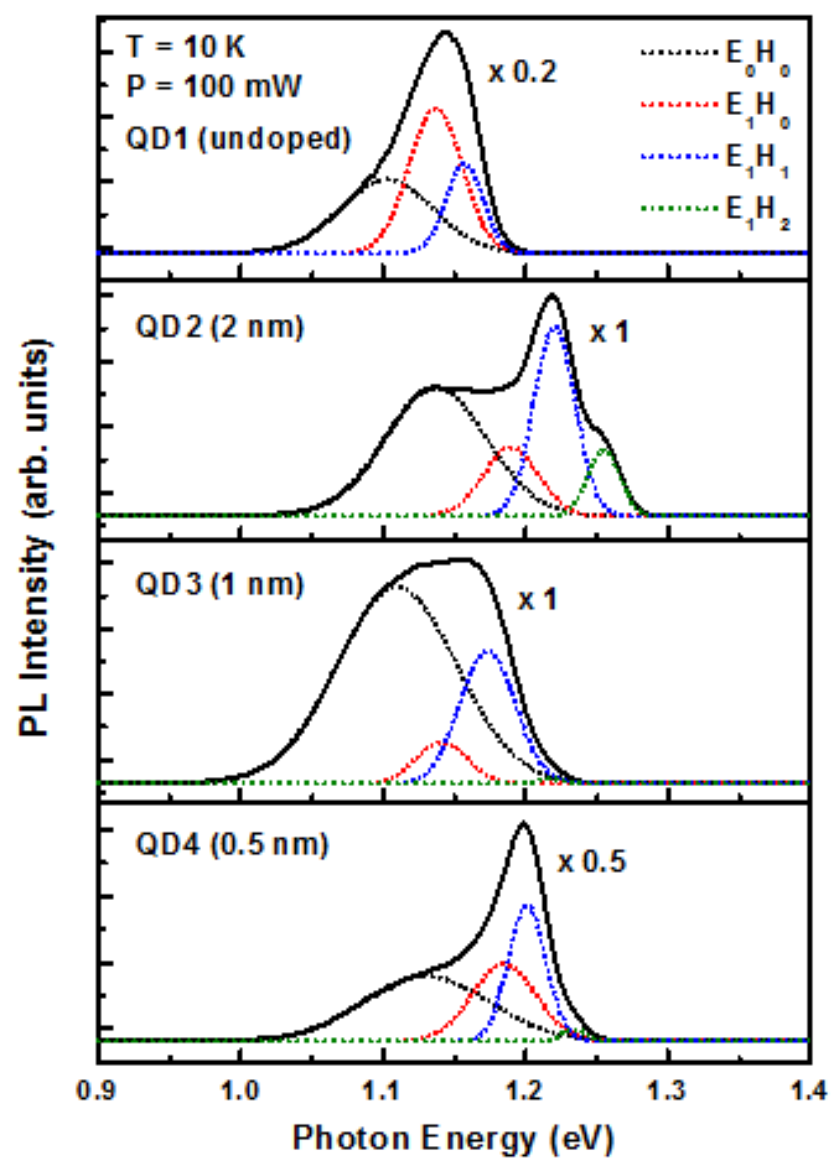

Figure 1. PL spectra taken from the undoped sample and doped samples with different doping position at $10 \mathrm{~K}$. Each PL spectrum is fitted with multiple Gaussian distribution functions. $\left(\mathrm{E}_{0}, \mathrm{E}_{1}\right.$ : electron ground and first excited state / $\mathrm{H}_{0}, \mathrm{H}_{1}, \mathrm{H}_{2}$ : hole ground, first, and second excited state). 

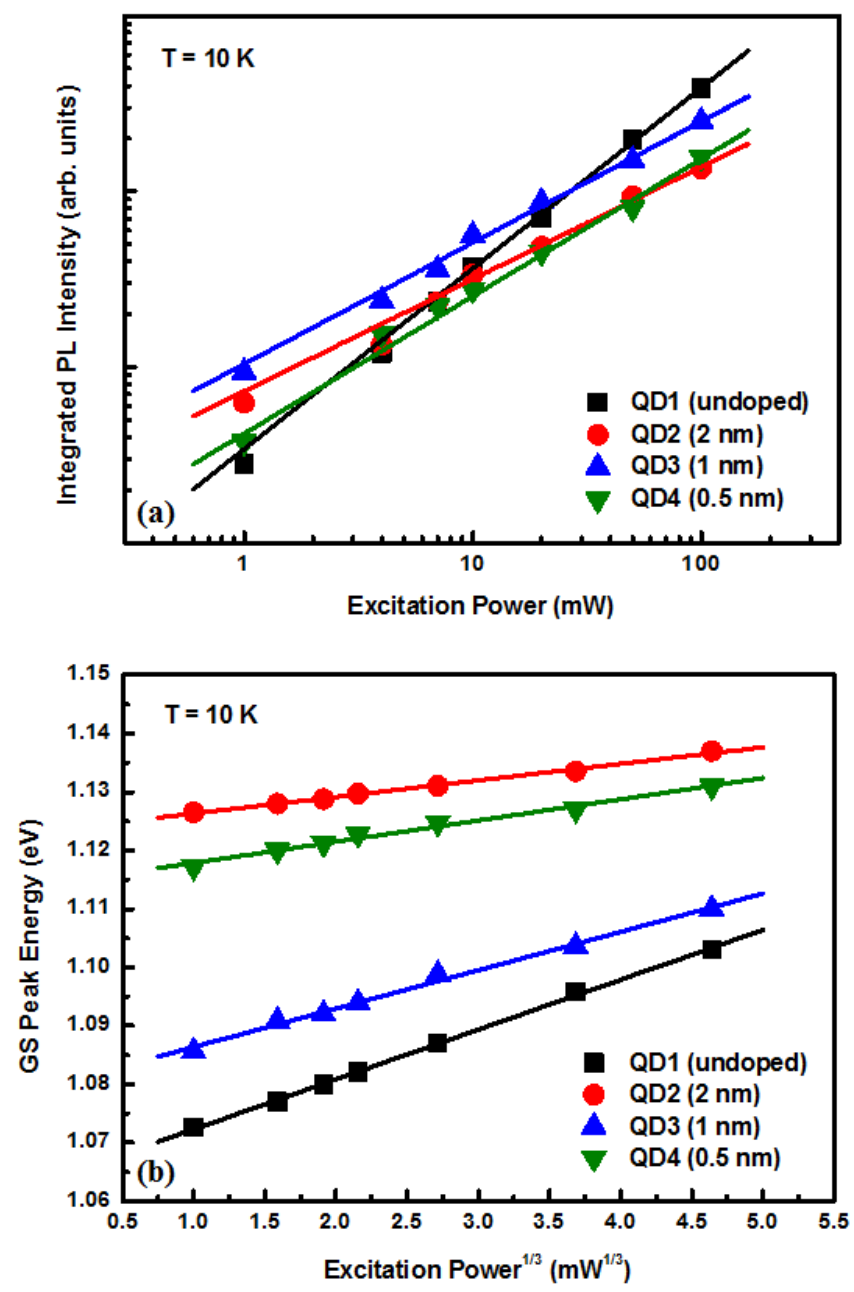

Figure 2. (a) Integrated PL intensities of the GS emissions against the excitation power at $10 \mathrm{~K}$ and (b) excitation power dependent GS peak shift of the undoped sample and doped samples at $10 \mathrm{~K}$. The solid linear lines in the figures are leastsquare fitted lines through the data points. 

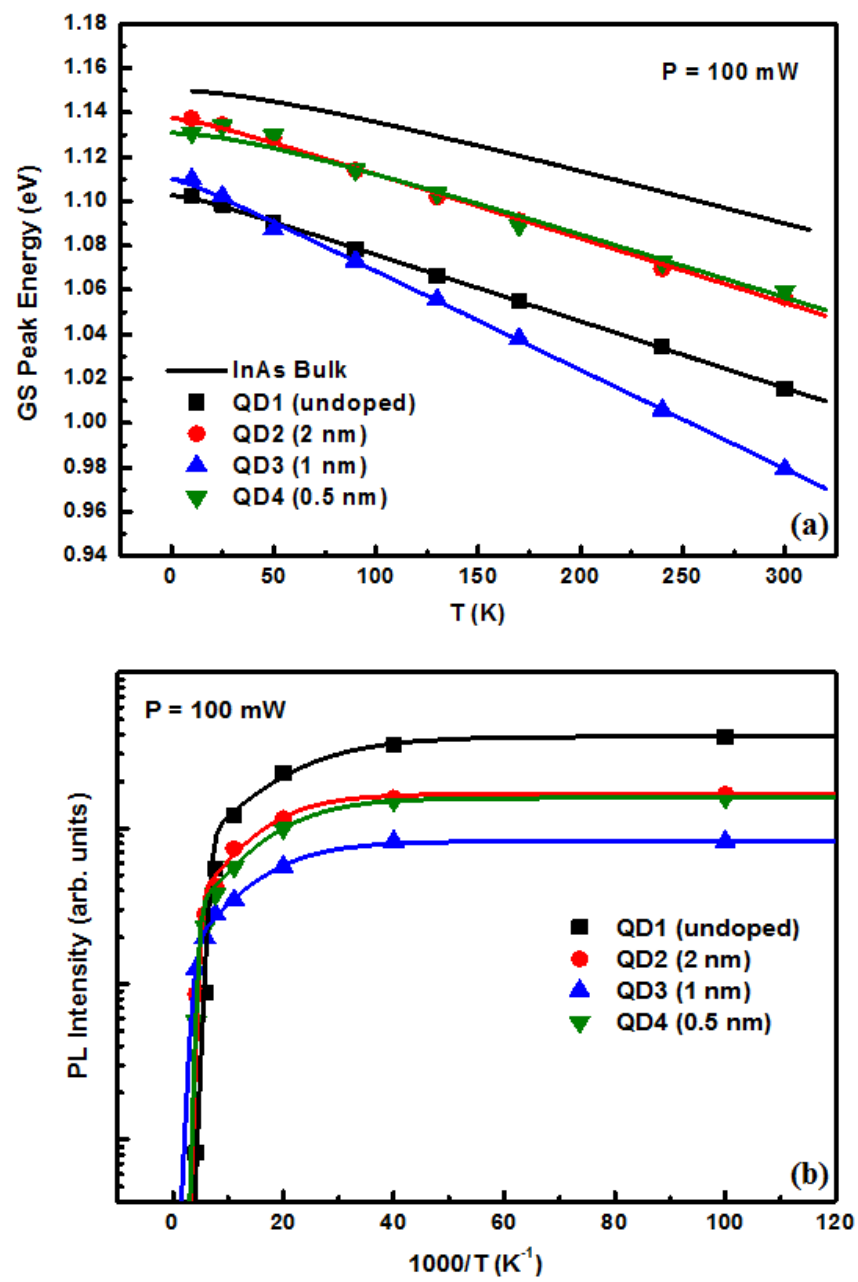

Figure 3. (a) Temperature-dependence of the GS PL peak energies and (b) Arrhenius plots of the integrated PL intensities of the GS emissions as a function of inverse temperature for the QD samples. 


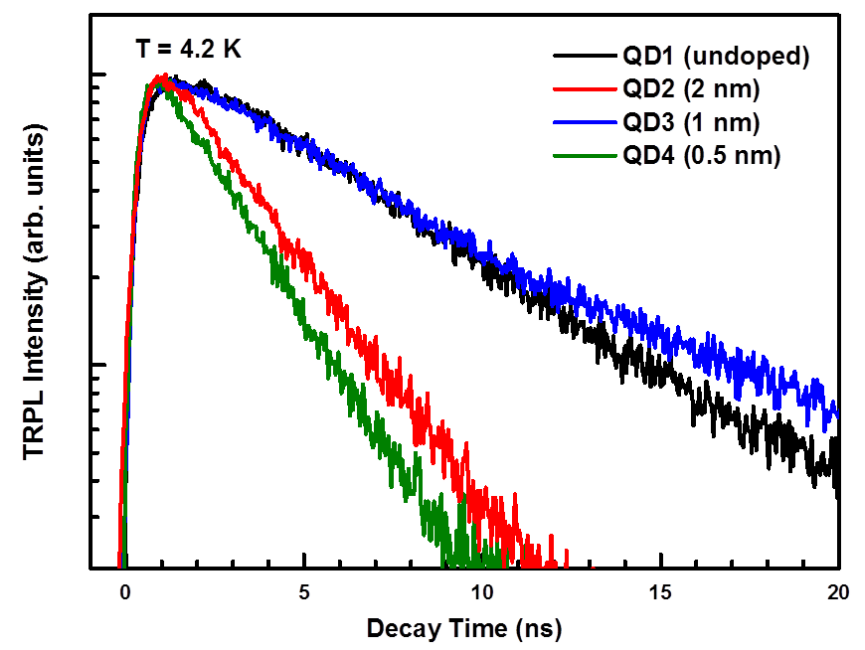

Figure 4. TRPL decay profiles measured at the GS emission peaks for the QD samples at $4.2 \mathrm{~K}$. 


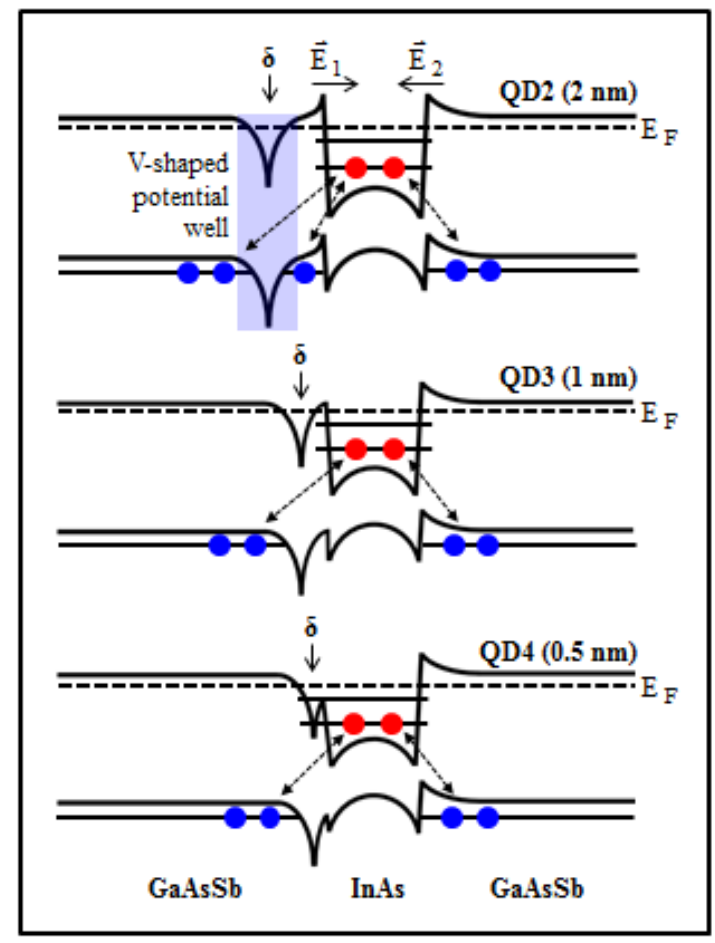

Figure 5. Schematic energy band diagrams of the doped samples with different doping positions. The dotted arrows indicate interband transitions between electrons confined in the QD potential and holes localized in the GaAsSb. The $\mathrm{E}_{1(2)}$ and $\mathrm{E}_{\mathrm{F}}$ denote a local electric field by the spatial separation of carriers and Fermi energy level, respectively. 
TABLE 1. TRPL carrier lifetimes and amplitude ratios extracted from the fitting for the undoped sample and doped samples at $4.2 \mathrm{~K}$.

\begin{tabular}{lcccc}
\hline \hline \multicolumn{1}{c}{ Sample } & $\tau_{1}(\mathrm{~ns})$ & $\tau_{2}(\mathrm{~ns})$ & $\tau_{\text {avg }}(\mathrm{ns})$ & $\mathrm{A}_{2} / \mathrm{A}_{1}$ \\
\hline QD1 (undoped) & 4.6 & 7.0 & 5.5 & 0.58 \\
QD2 $(2 \mathrm{~nm})$ & 2.2 & 3.2 & 2.5 & 0.39 \\
QD3 $(1 \mathrm{~nm})$ & 5.2 & 11.4 & 6.3 & 0.22 \\
QD4 $(0.5 \mathrm{~nm})$ & 1.9 & 3.0 & 2.1 & 0.26 \\
\hline
\end{tabular}

